\title{
Curonian Spit Biodiversity Plots
}

\section{Christian Dolnik}

\begin{abstract}
The Curonian Spit Biodiversity Plots database (GIVD ID EU-RU-009) was established in 2001 at the Ecology Centre of Kiel University in co-operation with the University of Kaliningrad. The database consists of 130 biodiversity plot series of nested plots from $0.0001 \mathrm{~m}^{2}$ to $900 \mathrm{~m}^{2}$ for 14 different vegetation types of the Curonian Spit Nationalpark. The focus was the census of vascular plants, bryophytes and lichens, including epiphytes for the analysis of species richness of different plant communities. Geobotanical relevés are available for the $900 \mathrm{~m}^{2}$ plots.
\end{abstract}

Keywords: plant community; nested plot; SAR; species richness.

\section{Curonian Spit Biodiversity Plots}

Scope: Analysis of the species-area-relationship of forest and grassland vegetation - a case study for measuring species diversity of vegetation including bryophytes and lichens

Status: finished Period: $1999-2001$

Database manager(s): Christian Dolnik (cdolnik@ecology.uni-kiel.de)

Owner: Christian Dolnik (private)

Web address: [NA]

Availability: according to a specific agreement

Online upload: [NA]

Online search: $[\mathrm{NA}]$

Database format(s): Excel

Export format(s): Excel

Publication: Dolnik C (2003): Artenzahl-Areal-Beziehungen von Wald- und Offenlandgesellschaften. Mitteilungen Arbeitsgemeinschaft Geobotanik Schleswig-Holstein Hambg., 62:183.

Plot type(s): nested plots

Plot-size range: $1-900 \mathrm{~m}^{2}$

Non-overlapping plots: 130

Total plot observations: 2,080

Estimate of existing plots: [NA]

Completeness: $[N A]$

Countries: RU: $100.0 \%$

Forest: [NA] - Non-forest: [NA]

Guilds: all vascular plants: 100\%; bryophytes (terricolous or aquatic): 100\%; lichens (terricolous or aquatic): 100\%; non-terricolous taxa (epiphytic, saxicolous, lignicolous): $100 \%$

Environmental data: soil depth: 100\%; surface cover other than plants (open soil, litter, bare rock etc.): $100 \%$; soil pH: $20 \%$

Performance measure(s): presence/absence only: $100 \%$; cover: $19 \%$

Geographic localisation: GPS coordinates (precision $25 \mathrm{~m}$ or less): $70 \%$

Sampling periods: $1990-1999: 23.0 \% ; 2000-2009: 77.0 \%$

Information as of 2012-07-12; further details and future updates available from http://www.givd.info/ID/EU-RU-009

Christian Dolnik (cdolnik@ecology.uni-kiel.de)

Ecology Centre Kiel, University of Kiel, Olshausenstr. 40, 24098 Kiel, GERMANY 